\title{
INTERACCIÓN GENOTIPO × AMBIENTE EN RENDIMIENTO DE PAPA (Solanum tuberosum L.) CON PULPA PIGMENTADA EN CUTERVO, PERÚ
}

\author{
GENOTYPE $\times$ ENVIRONMENT INTERACTION IN POTATO (Solanum \\ tuberosum L.) YIELD WITH PIGMENTED FLESH IN CUTERVO, PERU
}

\author{
Roberto Tirado M. ${ }^{1 *}$, Roberto Tirado L. ${ }^{2}$, y Juan Mendoza C. ${ }^{3}$ \\ Escuela de Agronomía, Universidad Nacional José Faustino Sánchez Carrión, Av. Mercedes Indaco- \\ chea $N^{\circ} 609$, Huacho, Perú. \\ 2 Escuela de Agronomía, Universidad Nacional Pedro Ruíz Gallo, Av. Juan XXIII Nº 391, Lambayeque, \\ Perú. \\ ${ }^{3}$ Escuela de Agronomía, Universidad Nacional Agraria La Molina, Av. La Molina s/n., Lima, Perú. \\ * Autor para correspondencia: hugotiradomalaver@gmail.com
}

\section{RESUMEN}

La interacción genotipo $\times$ ambiente estima el comportamiento diferencial que presentan los clones cuando se les evalúa en diferentes ambientes y permite determinar los clones con mayor estabilidad. El objetivo de la presente investigación fue estimar la magnitud de la interacción genotipo $\times$ ambiente para el rendimiento de catorce clones avanzados de papa con pulpa pigmentada y dos variedades comerciales (Amarilis y Canchán), en dos localidades productoras de papa en Cutervo, Perú, durante dos años. Para analizar los datos obtenidos se usó el análisis combinado de varianza y el modelo de efectos principales aditivos e interacción multiplicativa (AMMI). El análisis AMMI reveló que el efecto del ambiente, genotipo y la interacción genotipo $\times$ ambiente, explican el 13,15\%, 26,03\% y $60,82 \%$ de la suma de cuadrados total en el rendimiento de tubérculos de papa, respectivamente; por tanto, la mayor parte fue aportada por el efecto de la interacción genotipo-ambiente. Los primeros dos componentes principales fueron altamente significativos $(\mathrm{p}<0,01)$ y capturaron el $45,58 \%$ y $38,61 \%$ de la suma de cuadrados total de la interacción genotipo $\times$ ambiente en el rendimiento. El biplot muestra a los clones CIP302304.15, CIP302295.32 y Amarilis INIA registraron valores de 980,64, 959,86 y 1001,35 g planta $^{-1}$, respectivamente, con rendimiento superior a la media general y una baja interacción con los ambientes evaluados, mostrando una mayor estabilidad de rendimiento de papa con pulpa pigmentada.

Palabras clave: análisis AMMI, clones, componentes principales, estabilidad, suma de cuadrados.

\begin{abstract}
Genotype $\times$ environment interaction estimates the differential behavior of clones when evaluated in different environments, and it allows the identification of stable clones. The objective of this study was to estimate the magnitude of the genotype $\times$ environment interaction for the yield of fourteen advanced potato clones with pigmented flesh and two commercial varieties (Amarilis and Canchán), in two potato growing locations in Cutervo, Peru, over a period of two years. Data were analyzed using the combined analysis of variance and additive main effects and multiplicative interaction (AMMI) model. The AMMI analysis revealed that the effect of the environment, genotype and genotype environment interaction explains $13.15 \%, 26.03 \%$ and $60.82 \%$ of the total sum of squares in
\end{abstract}

Recibido: 27 abril $2018 . \quad$ Aceptado: 27 julio 2018. 
the yield of potato tubers, respectively; therefore, the greatest contribution corresponded to the effect of the genotype $\times$ environment interaction. The first two main components were highly significant $(\mathrm{p}<0.01)$ and captured $45.58 \%$ and $38.61 \%$ of the total sum of squares of the genotype-environment interaction in the yield. The biplot shows that the clones CIP302304.15, CIP302295.32 and Amarilis INIA recorded values of $980.64,959.86$ and 1001.35 g plant $^{-1}$, respectively, with a higher yield compared to the average value and a low interaction with the environments evaluated, indicating greater yield stability in potato with pigmented flesh.

Key words: AMMI analysis, clones, main components, stability, sum of squares.

\section{INTRODUCCIÓN}

La papa (Solanum tuberosum L.) es el cultivo alimenticio no cereal más consumido del mundo y está clasificado como el cuarto cultivo más importante después del arroz, el trigo y el maíz (Zhang et al., 2017). El aumento de la conciencia sobre la salud del corazón y la prevención de enfermedades ha llevado a los consumidores a elegir alimentos más proactivos (Bach et al., 2012). Los tubérculos con pulpa pigmentada presentan diferentes colores de pulpa (violeta, rojo, azul, negro, crema y bicolor). Aquellas variedades pigmentadas tienen un elevado contenido de vitamina $\mathrm{C}$ y antocianinas naturales, las cuales tienen propiedades antioxidantes, que poseen beneficios potenciales para la salud (Landrum et al., 2001). Además, le confieren mecanismos de defensa contra la acción de los radicales libres de oxígeno (ROS) que generan problemas de estrés oxidativo, provocando así, enfermedades cancerígenas, cardiovasculares, entre otras (Imeh y Khokhar, 2002; Cano y Arnao, 2004).

La interacción genotipo $\times$ ambiente (IGA), se refiere a la respuesta diferencial de los genotipos evaluados en diferentes condiciones ambientales (Tonk et al., 2011). Es un fenómeno complejo, ya que involucra condiciones ambientales (agroecológicas, climáticas y agronómicas) y todos los factores fisiológicos y genéticos que determinan el crecimiento y desarrollo de las plantas (Mohammadi et al., 2016). Es así que el amplio rango de ambientes climáticos y edáficos en los que se cultivan, pueden provocar respuestas diferenciales del comportamiento de los genotipos en los diferentes ambientes como resultado de la IGA (Abbott y Pistorale, 2011).

En el mejoramiento genético de plantas, el estudio de la IGA es esencial para la eficiencia del proceso selectivo, debido a que la mayoría de los caracteres de importancia agronómica son de herencia cuantitativa, como el rendimiento, el cual es un carácter que presenta distribución continúa, posee herencia poligénica y es muy influenciado por las variaciones del ambiente (Chaves, 2001, citado por Abbott y Pistorale, 2011).

La magnitud de la IGA del rendimiento es un resultado combinado de los efectos del genotipo, el medio ambiente y la IGA, siendo el genotipo y la IGA los más relevantes para la evaluación de variedades y la identificación de mega-ambientes (Yan et al., 2000). Al analizar la IGA, este permite reportar información necesaria para identificar genotipos estables de alto rendimiento y con rasgos deseables, para recomendar estos nuevos genotipos para su liberación como variedad comercial (Lule et al., 2015).

La baja productividad del cultivo de papa podría atribuirse principalmente a la falta de variedades estables de alto rendimiento y adaptables a los ambientes adversos, a las malas prácticas de manejo y otros factores bióticos y abióticos (Bezawuletaw et al., 2006, citado por Lule et al., 2015). Además, los agricultores necesitan variedades que muestren un alto rendimiento, sin embargo, la limitante en la selección de variedades es la alta IGA del rendimiento. Para superar el problema de IGA, los ensayos generalmente se llevan a cabo en varias localidades y años, para garantizar que los genotipos seleccionados tengan un rendimiento alto y estable en una amplia gama de ambientes (Gedif y Yigzaw, 2014).

El análisis de IGA se realiza utilizando diferentes métodos estadísticos, entre ellos, el más utilizado en la actualidad es el modelo de efectos principales aditivos e interacción multiplicativa AMMI, el cual demostró ser una herramienta estadística valiosa (Affleck et al., 2011). El modelo permite la interpretación del efecto de la IGA en los ensayos de evaluación, siendo estos ensayos las que proporcionan información esencial para seleccionar y recomendar variedades estables (Yan y Tinker, 2006; Asnake et al., 2013).

El modelo AMMI combina el análisis de varianza (ANDEVA), que estudia el efecto de los genotipos y el medio ambiente, con el análisis de componentes principales, que estudia el efecto de la IGA, de forma multivariada y permite visualizar el comportamiento de los genotipos y ambientes de evaluación mediante el gráfico biplot (Vargas y Crossa, 2000). Entonces los componentes principales obtenidos por el AMMI y el biplot ayudan a los fitomejoradores a explicar la IGA permitiendo identificar y seleccionar genoti- 
pos prometedores que rinden mejor en términos de potencial de rendimiento, además de poseer un rendimiento estable bajo condiciones ambientales específicas (Adebola et al., 2013). Teniendo en cuenta que para elegir genotipos superiores, debe existir una baja o mínima IGA (Cotes et al., 2002). Es decir, los genotipos con valores cercanos al origen del primer componente principal en el gráfico biplot, presentan una baja interacción con los ambientes evaluados, siendo los más estables y al alejarse del origen su interacción con los ambientes es mayor (Yan et al., 2000).

Por lo tanto, los fitomejoradores se preocupan principalmente por las variedades estables y de alto rendimiento, ya que el desarrollo de variedades es un esfuerzo que lleva mucho tiempo. Siendo nuestro principal interés desarrollar y obtener nuevas variedades de papa con alto potencial de rendimiento (Cotes et al., 2002).

El objetivo de este estudio fue estimar la magnitud de IGA para el rendimiento de tubérculos de clones avanzados de papa y seleccionar los clones con mayor estabilidad de rendimiento de tubérculos con pulpa pigmentada en Cutervo, Perú.

\section{MATERIALES Y MÉTODOS}

La presente investigación se desarrolló en las zonas productoras de papa en la provincia de $\mathrm{Cu}-$ tervo, Cajamarca, ubicada a $6^{\circ} 22^{\prime} 53^{\prime \prime}$ de Latitud Sur y $78^{\circ} 49^{\prime} 06^{\prime \prime}$ de Longitud Oeste, a una altitud de 2.617 msnm, Perú. La evaluación del material experimental se realizó en la localidad de Chaquil durante dos años (2015-2016 y 2016-2017) y en la localidad de Huichud durante dos años (20152016 y 2016-2017). Cabe resaltar que cada ensayo realizado en una localidad y año representó un ambiente de evaluación, lo cual generó un total de cuatro ambientes evaluados.

En cada ambiente se realizó un diseño experimental de bloques completos al azar con tres repeticiones. La parcela experimental fue de 30 plantas ( 3 surcos de 10 plantas cada uno), siendo considerado el surco central para las evaluaciones. El distanciamiento entre surcos y entre plantas fue de 1 metro $\times 30 \mathrm{~cm}$, respectivamente.

\section{Material vegetal}

Se evaluaron catorce genotipos avanzados mejorados de pulpa pigmentada procedentes del Centro Internacional de la Papa (CIP): “CIP 302281.17 (Clon1), CIP 302298.44 (Clon2), CIP 302295.32 (Clon3), CIP 302285.27 (Clon4), CIP 302281.52 (Clon5), CIP 302290.13 (Clon6), CIP 302288.14 (Clon7), CIP 302297.35 (Clon8), CIP 302286.13 (Clon9), CIP 302288.39 (Clon10), CIP 302281.39 (Clon11), CIP 302304.15 (Clon12), CIP
302298.42 (Clon13), CIP 302284.17 (Clon14)" y dos variedades comerciales 'Amarilis INIA (VC15)' y 'Canchan INIA (VC16)'.

\section{Análisis estadístico}

En cada experimento se realizó el respectivo análisis de variancia y las medias fueron comparadas por la prueba de Tukey a un nivel de $5 \%$ de probabilidad. El modelo aditivo lineal a emplear fue:

$$
Y i j k=\mu+\beta i+T j+E i j
$$

dónde: Yijk = ijk-ésima observación del i-ésimo bloque; el j-ésima variedad y K-ésimo nivel, $\mu=$ media general, $\beta \mathrm{i}=$ efecto $\mathrm{i}$-ésima de bloque, $\mathrm{T} j=$ efecto j-ésimo tratamiento, Eijk = efecto aleatorio del error.

Para el análisis de estabilidad fenotípica se utilizó el Modelo de efectos principales aditivos e interacción multiplicativa (Modelo AMMI), el cual explica inicialmente los efectos principales de genotipos y ambientes mediante un análisis de varianza convencional y posteriormente describe la parte no aditiva correspondiente a la IGA por medio de un análisis multivariado de componentes principales (CPl, $\mathrm{CP} 2, \mathrm{CPn})$. A partir del primer componente principal y el resto de los componentes, en caso de representar un porcentaje aceptable de la interacción, el modelo AMMI genera un gráfico de dos dimensiones (biplot), en el que se pueden observar las diferencias entre ambientes, el grado de interacción de los genotipos con el ambiente, la estabilidad y las adaptaciones específicas de algunos genotipos a determinados ambientes (Vargas y Crossa, 2000). El modelo AMMI se analizó mediante el programa propuesto por Vargas y Crossa (2000) quienes forman parte del modelo de Zobel (1988), que será representado mediante la siguiente fórmula matemática:

$$
Y_{i j}=\mu+g_{i}+e_{j}+\sum_{k=1}^{p} \lambda_{k} \gamma_{i k} \alpha_{j k}+\varepsilon_{i j}
$$

dónde: $Y i j=$ es el rendimiento del i-ésimo genotipo en el j-ésimo ambiente, Los parámetros aditivos son: $\mu=$ media genera; $\mathrm{Gi}=$ Efecto del i-ésimo genotipo; $\sigma j=$ Efecto del j-ésimo ambiente; $\lambda \mathrm{k}=$ Valor propio del componente principal K, $\alpha \mathrm{ij}{ }^{*} \mathrm{yjk}$ $=$ Valor del componente principal $\mathrm{k}$ de genotipo $\mathrm{y}$ localidad; $\varepsilon \mathrm{ij}=$ Error experimental.

El análisis estadístico combinado y AMMI fueron realizados con el paquete estadístico SAS (2003).

\section{RESULTADOS Y DISCUSIÓN}

\section{Análisis de varianza de AMMI}

El análisis de varianza de efectos principales y aditivos (AMMI) para la fuente de genotipos, 
ambientes y la IGA fueron altamente significativas $(\mathrm{P}<0,001)$ (Tabla 1). Este resultado indica la presencia de variación sustancial entre los clones sobre los ambientes donde se desarrollan (Maharana, 2017). Resultados similares concuerdan con los obtenidos por Gedif y Yigzaw (2014), quienes evaluando el efecto de la IGA para la estabilidad de rendimiento de ocho clones avanzados de papa, lograron detectar diferencias para los efectos principales de genotipos, ambientes y la IGA.

Los resultados también mostraron que el $60,82 \%$ suma de cuadrados total del rendimiento de tubérculos $\left(\mathrm{g}_{\text { plant }}{ }^{-1}\right.$ ) fue atribuible por el efecto de la IGA, seguido por el efecto genético y ambiental los cuales explicaron $13,15 \%$ y $26,03 \%$ de la variación total de la suma de cuadrados, respectivamente. La mayor parte de la suma de cuadrados total es aportada por la IGA, lo que implica que el rendimiento está influenciado por factores genéticos, factores ambientales y principalmente por su interacción (Affleck et al., 2011; Adebola et al., 2013). Los resultados obtenidos fueron similares con los obtenidos por Muthoni et al. (2015) quienes, evaluando el efecto de la IGA y estabilidad de rendimiento de 48 familias de papa, mostraron que la contribución del efecto de la IGA a la variación del rendimiento fue del $43,42 \%$, mientras que la de genotipos fue del $33,15 \%$ y los ambientes del $23,43 \%$. Los resultados también concuerdan con Iragaba (2014), quien evaluando la estabilidad de rendimiento de once clones avanzados de papa, encontró que el efecto de la IGA superó a los efectos del genoti- po y del ambiente en la suma de cuadrados de la varianza total.

La prueba de promedios de Tukey al 5\% para el análisis AMMI (Tabla 2) encontró que existe suficiente evidencia estadística, para afirmar que la constitución genética de cada genotipo influye en el rendimiento. Así también, el efecto que tiene el medio ambiente en la variación de estos genotipos debido al carácter poligénico que rige esta característica. Esta prueba estableció tres rangos de significación estadística, compartiendo el primer rango; se encontraron ocho genotipos: CIP 302281.17, CIP 302298.44 a CIP 302304.15 que sobresalen con mayor rendimiento, con promedios de 1.124,54, 1.115,62, a 980,54 g planta $^{-1}$. En el segundo grupo se ubicaron los genotipos: CIP 302295.32 y Canchan INIA con promedios que oscilan entre 959,86 y 879,23 g planta $^{-1}$. En el último rango se encontraron cuatro genotipos: CIP 302286.13 con 879,06 g planta $^{-1}$ al CIP 302281.39 con 787,40 g planta $^{-1}$, respetivamente.

\section{Análisis de estabilidad}

Los resultados de los análisis de estabilidad se presentan en la Tabla 1 y Fig. 1. Al descomponer la suma de cuadrados de la IGA en componentes principales $(\mathrm{CP})$; se constató que los dos primeros ejes fueron altamente significativos $(\mathrm{P}<0,001)$, los cuales explicaron $84,19 \%$ de la variabilidad total de la IGA. El primer eje del componente principal (CP1) explicó el 45,58\% de la suma de cuadrados de IGA, mientras que el segundo eje de componente principal (CP2) solo explicó el 38,61\% de

Tabla 1. Análisis de varianza AMMI y significancia estadística del rendimiento (g planta $\left.{ }^{-1}\right)$ de catorce genotipos avanzados de papa con pulpa pigmentada y dos variedades comerciales evaluados en cuatro ambientes de Cutervo, Perú.

Table 1. AMMI variance analysis and statistical significance of the yield $\left(\mathrm{g} \mathrm{plant}^{-1}\right)$ of fourteen advanced potato genotypes with pigmented flesh and two commercial varieties evaluated in four environments of Cutervo, Peru.

\begin{tabular}{lrrccc}
\hline Fuente de Variación & GL & \multicolumn{1}{c}{ SC } & CM & SC (\%) & Acumulado SC (\%) \\
\hline Ambientes & 3 & $933.292,48$ & $202.963,08^{* *}$ & 13,15 & 13,15 \\
Genotipos & 15 & $1.847 .869,97$ & $123.191,33^{* *}$ & 26,03 & 39,18 \\
Interacción G × A & 45 & $4.317 .298,21$ & $95.939,96^{* *}$ & 60,82 & 100,00 \\
CP1 & 17 & $5.903 .599,14$ & $347.270,54^{* *}$ & 45,58 & 45,58 \\
CP2 & 15 & $5.000 .682,06$ & $333.378,80^{* *}$ & 38,61 & 84,19 \\
CP3 & 13 & $2.047 .613,46$ & $157.508,73 N S$ & 15,81 & 100,00 \\
Error & 120 & $1.864 .801,79$ & $15.540,02$ & & \\
Total & 191 & $8.963 .262,47$ & & & \\
\hline
\end{tabular}

$\mathrm{GL}$ = grados de libertad; $\mathrm{SC}=$ suma de cuadrados; $\mathrm{CM}=$ cuadrados medios; $\mathrm{CV}=$ coeficiente de variación; $\mathrm{CP} 1=$ componente principal $1 ; \mathrm{CP} 2=$ componente principal 2.

$\mathrm{NS},{ }^{*}, * *$ : no significativo, y significativo a $\mathrm{P} \leq 0,05$ y 0,01 , respectivamente.

$\mathrm{DF}=$ degrees of freedom; $\mathrm{SS}=$ sum of squares; $\mathrm{SM}=$ square means; $\mathrm{CV}=$ coefficient of variation; $\mathrm{MC} 1=$ main component $1 ; \mathrm{MC} 2=$ main component 2 .

NS, ${ }^{*}{ }^{* *}$ : not significant and significant at a $\mathrm{P} \leq 0.05$ and 0.01 , respectively. 
Tabla 2. Rendimiento de tubérculos y contribución de los dos primeros componentes principales del modelo AMMI.

Table 2. Yield of tubers and contribution of the first two main components of the AMMI model.

\begin{tabular}{lcccr}
\hline Factores & & Rdto. $\left(\right.$ g planta $\left.^{-1}\right)$ & CP1 & CP2 \\
\hline Genotipos & CIP 302281.17 & $1.124,54 \mathrm{a}$ & $-6,6738$ & $-1,9410$ \\
& CIP 302298.44 & $1.115,62 \mathrm{a}$ & $-7,1626$ & 7,3176 \\
& CIP 302285.27 & $1.051,98 \mathrm{ab}$ & 0,7948 & $-6,4831$ \\
& CIP 302298.42 & $1.044,04 \mathrm{ab}$ & 8,8558 & 0,1163 \\
& Amarilis INIA & $1.001,35 \mathrm{abc}$ & 1,2747 & 5,0569 \\
& CIP 302290.13 & $993,83 \mathrm{abc}$ & $-5,673$ & $-5,3984$ \\
& CIP 302288.14 & $980,64 \mathrm{abcd}$ & $-9,1947$ & 4,4645 \\
& CIP 302304.15 & $980,54 \mathrm{abcd}$ & 2,7458 & $-1,2035$ \\
& CIP 302295.32 & $959,86 \mathrm{bcde}$ & $-0,9271$ & $-3,9976$ \\
& CIP 302297.35 & $954,91 \mathrm{bcde}$ & $-6,5249$ & 9,1302 \\
& CIP 302281.52 & $917,86 \mathrm{bcde}$ & $-12,7591$ & $-5,3984$ \\
& Canchan INIA & $879,23 \mathrm{bcde}$ & 17,2891 & $-3,5448$ \\
& CIP 302286.13 & $879,06 \mathrm{bcde}$ & 0,5168 & $-6,0553$ \\
& CIP 302284.17 & $835,34 \mathrm{cde}$ & 9,4505 & 1,4190 \\
& CIP 302288.39 & $807,04 \mathrm{de}$ & 8,0704 & 8,7568 \\
Ambientes & CIP 302281.39 & $787,40 \mathrm{e}$ & 7,9641 & $-6,3222$ \\
& Ambiente 1 & 981,35 & $-2,5878$ & 23,4971 \\
& Ambiente 2 & 862,48 & $-17,2637$ & $-10,1856$ \\
& Ambiente 3 & 973,87 & 22,3382 & $-5,9673$ \\
& Ambiente 4 & $1.010,61$ & $-2,4866$ & $-7,3443$ \\
\hline
\end{tabular}

Rdto. $=$ Promedio del rendimiento de tubérculos; $\mathrm{CP} 1=$ componente principal $1 ; \mathrm{CP} 2=$ componente principal 2. Rdto. $=$ Average yield of tubers; CP1 = main component $1 ; \mathrm{CP} 2=$ main component 2.

la suma de cuadrados de IGA. Por lo que el CP1 constituye un parámetro válido para el análisis de estabilidad del rendimiento de tubérculos ( $\mathrm{g}$ planta $\left.^{-1}\right)$. Resultado similar fue obtenido por Salomón et al. (2015), quienes analizando la IGA de trece progenies de papa y tres cultivares testigos, indicaron que la partición de IGA a través del análisis AMMI mostró que el CP1 y CP2 representaron el $44,17 \%$ y el $25,83 \%$ de la suma de cuadrados de IGA, respectivamente, lo que explica un total de variación del 70,00 \%.

En la Tabla 2 se presentan los valores escalares de los componentes principales y la media para cada ambiente y genotipo, el signo y la magnitud del valor indican la interacción y el sentido de la misma, mostrándose en la Fig. 1. En la representación de los efectos de la IGA (Fig.1), los genotipos que tienen puntajes de $\mathrm{CP} 1>0$, tienden a dar un rendimiento promedio más alto y los que tienen puntajes de $\mathrm{CP} 1<0$, se identificaron como de menor rendimiento (Yan y Tinker, 2006). Los genotipos que reportan valores cercanos al origen del CP1, tienen menor magnitud en la interacción. Así los genotipos cercanos al origen son los más estables, y al alejarse de éste, su comporta- miento es más variable (Yan et al., 2000).

Los clones Clon13, Clon8, Clon7, Clon12, Clon6, Clon15, Clon4, Clon13, Clon2 y Clon1 se identificaron como de alto rendimiento $(\mathrm{CP} 1>0)$, mientras Clon5, Clon9, Clon16, Clon14, Clon10 y Clon11 como clones de bajo rendimiento $(\mathrm{CP} 1<$ 0) (Fig. 1).

El "biplot" de la Fig. 1 muestra a los clones CIP 302295.32 (Clon3), CIP 302304.15 (Clon 12) y Amarilis INIA (VC15) con valores cercanos al origen, indicando una baja IGA y con rendimiento superior a la media general, mostrando mayor estabilidad de rendimiento en los ambientes evaluados. Los clones CIP 302281.17 (Clon1) y CIP 302298.44 (Clon2) presentaron los rendimientos más altos, pero tuvieron mayor interacción con los ambientes evaluados. El clon CIP 302281.52 (Clon 5), CIP 302281.39 (Clon11) y CIP 302288.39 (Clon10) mostraron una alta IGA y un rendimiento inferior a la media general.

La nueva variedad desarrollada debe tener una amplia estabilidad de rendimiento en una amplia gama de ambientes, además de un alto potencial de rendimiento (Adebola et al., 2013; Mohammadi et al., 2016). Siendo el clon CIP 302295.32 


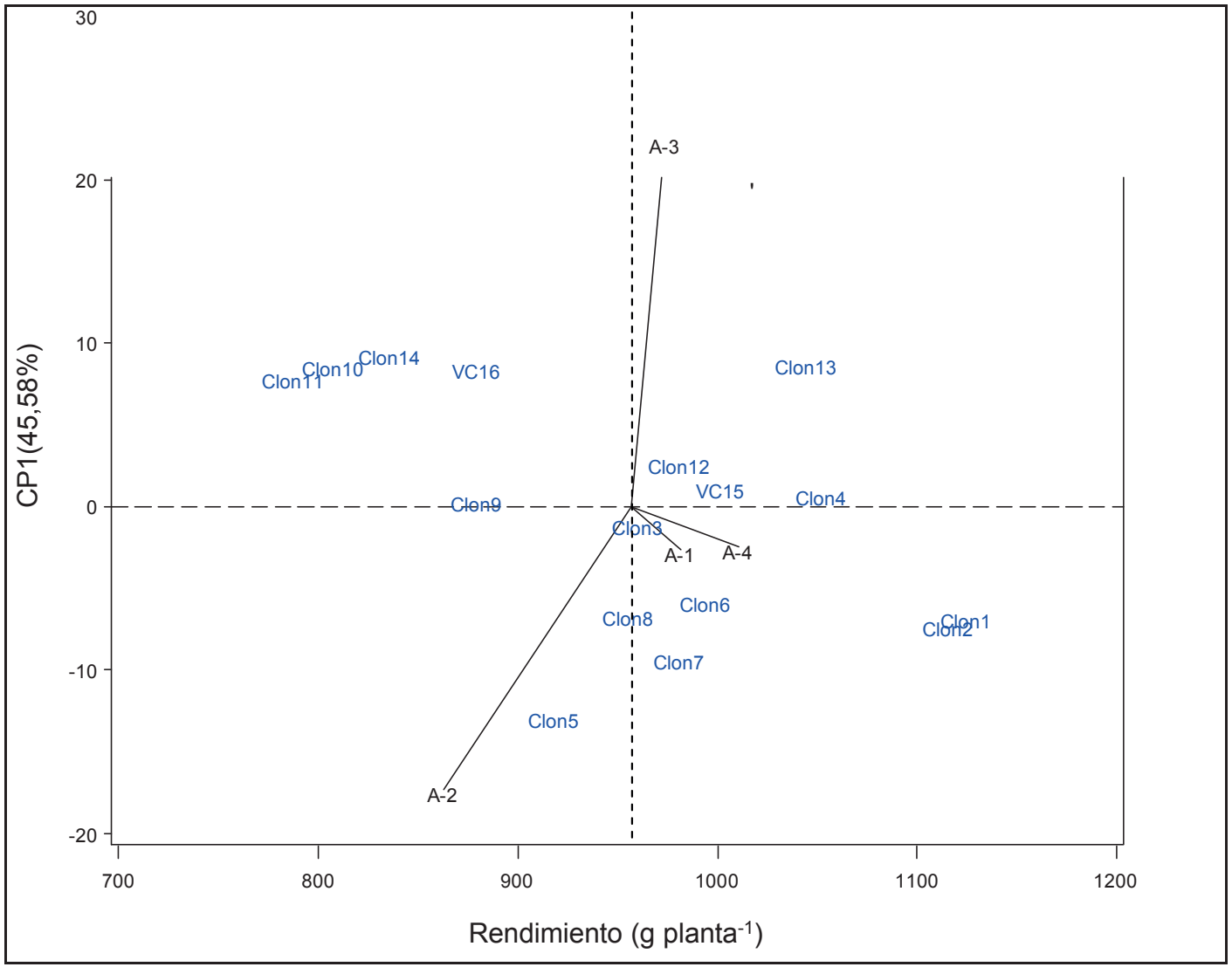

Fig. 1. Gráfica biplot del rendimiento $\left(\mathrm{g}_{\text {planta }}{ }^{-1}\right.$ ) y coeficientes del CP1 para la interacción de catorce genotipos avanzados de papa con pulpa pigmentada y dos variedades comerciales evaluados en cuatro ambientes de Cutervo, Perú.

Fig. 1. Biplot plot of yield $\left(\mathrm{g} \mathrm{plant}^{-1}\right)$ and PC1 coefficients for the interaction of fourteen advanced potato genotypes with pigmented flesh and two commercial varieties evaluated in four environments of Cutervo, Peru.

con 959,86 g planta $^{-1}$, el que registró mayor estabilidad y buen rendimiento en los ambientes evaluados.

El biplot ayuda a visualizar el comportamiento de la IGA, a través de la longitud de los vectores, que tienen la capacidad de discriminación de los ambientes (Yan y Tinker, 2006).

El ambiente 4 (A-4) presentó un valor bajo en el CP1 y mostró rendimiento superior a la media general, mientras que el ambiente 1 (A-1) detectó valores bajos en el $\mathrm{CP} 1$, pero el rendimiento fue menor a la media general. En tanto, el ambiente 3 (A-3) mostró valores altos en el CP1 y rendimiento superior a la media general, sin embargo, el ambiente 2 (A-2) presentó alto valor en el CP1 y rendimientos inferiores a la media general. Siendo estos ambientes los que mejor discriminaron a los clones en la evaluación, en cambio los ambientes A-1 y A-4 no discriminaron bien entre clones.

El mejor genotipo para el ambiente A-1 fue el clon CIP 302295.32, para el A-2 el CIP 302281.52, en tanto los altos rendimientos obtenidos en la localidad de Chaquil (A-1 y A-2) fueron resultado de los elementos climáticos apropiados para el desarrollo del cultivo, así como la precipitación pluvial con una buena distribución de lluvia durante el crecimiento y desarrollo de la planta, temperaturas bajas pero soportables por los genotipos, lo que permitió una eficiente fotosíntesis y distribución de fotosintatos lo que generó un aumento del número de gránulos de almidón como consecuencia alta producción. No obstante, que dichos suelos presentaron buena fertilidad, apropiada materia orgánica y $\mathrm{pH}$ casi neutral, siendo factores que influyeron en la respuesta positiva del genotipo.

En la localidad de Huichud (A-3 y A-4), las intensas precipitaciones pluviales durante el periodo lluvioso, permitió una buena humedad del suelo, incluyendo también temperaturas óptimas 


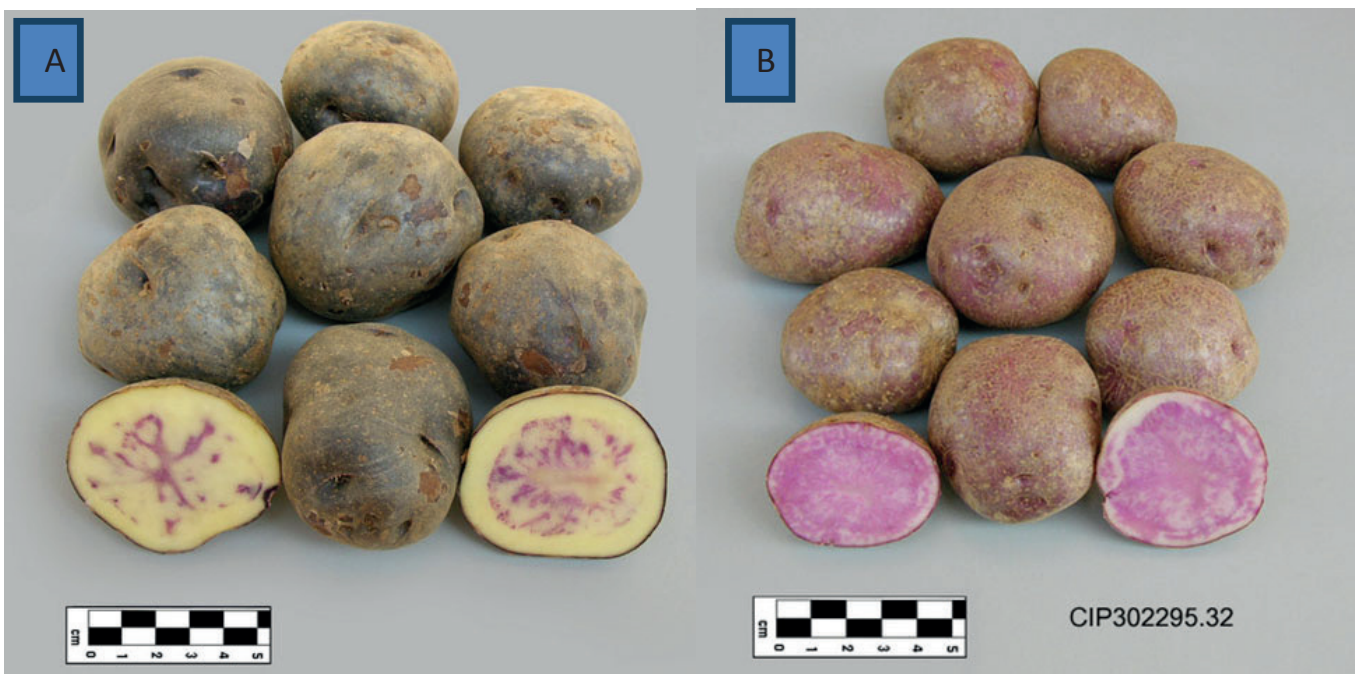

Fig. 2. Clones seleccionados por su alta estabilidad y rendimiento superior a la media general: (A) CIP302304.15; (B) CIP302295.32.

Fig. 2. Clones selected for their high stability and performance above the average: (A) CIP302304.15; (B) CIP302295.32.

lo que permitió un buen desarrollo de la planta, cabe resaltar que en el A-1 se presentó una helada (baja temperatura por debajo de la temperatura mínima anual) en el mes de junio lo que originó un bajo rendimiento de algunos genotipos, sin embargo, un buen número de genotipos presentaron buena producción de tubérculos. Aunado a la anterior, aquel suelo presentó buena fertilidad, apropiada materia orgánica y $\mathrm{pH}$ casi neutral, siendo factores que influyeron en la respuesta positiva del genotipo.

Bonierbale et al. (2004), mencionan que el desarrollo de genotipos que muestran adaptación a ambientes heterogéneos teniendo diferencias en altitud, precipitación, temperatura y tipo de suelo que predominan entre ambientes, llegan a obtener mayor estabilidad en ambientes marginales lo cual permite reducir la cantidad de insumos usados en el proceso productivo.

\section{CONCLUSIONES}

La contribución de la IGA a la variación total del rendimiento es de $60,82 \%$, mientras que el efecto del genotipo es de $26,03 \%$ y el ambiente $13,15 \%$.

Al particionar la IGA en $\mathrm{CP}$, los primeros dos CP explican el 84,19\% de la variabilidad total causada por la IGA. De los cuales, el CP1 y CP2 representan el 45,58 \% y 38,61 \%, respectivamente.

El rendimiento promedio y la estabilidad del biplot de los clones probados en los ambientes de prueba indican que CIP 302295.32 (Clon3), CIP 302304.15 (V-12) y la variedad comercial Amarilis
INIA (V-15) tienen un rendimiento superior a la media general, como también mayor estabilidad.

Por lo tanto, en base en el alto potencial de rendimiento y la estabilidad en los ambientes de prueba, se concluye que el clon CIP 302295.32 (V$3)$ es el mejor clon para los ambientes de evaluación.

\section{AGRADECIMIENTOS}

Se agradece al Centro Internacional de la Papa (CIP) por el material genético usada en nuestra investigación, a la Convención No. 176-FINCyT-FIDECOM-PIPEI-2012 y a la Asociación para la Conservación y Mejoramiento de la Biodiversidad Altoandino -ACOMEBIO.

\section{LITERATURA CITADA}

Abbott, L., y S. Pistorale. 2011. Análisis de la estabilidad y adaptabilidad de caracteres de interés agronómico en genotipos selectos de cebadilla criolla (Bromus catharticus). Agriscientia 28(2):109-117.

Adebola, P.O., A.G. Shegro, S.M. Laurie, L.N. Zulu, and M. Pillay. 2013. Genotype x environment interaction and yield stability estimate of some sweet potato [Ipomoea batatas (L.) Lam] breeding lines in South Africa. J. Plant Breed. Crop Sci. 5:182-186.

Affleck, J. J.A. Sullivan, Tarn, R., and D.E. Falk. 2011. Genotype by environment interaction effect on yield and quality of potatoes. Can. J. Plant Sci. 88:1099-1107. 
Asnake W.M. Henry, Z. Temesgen, and T. Girma. 2013. Additive main effects and multiplicative interactions model (AMMI) and genotype main effect and genotype by environment interaction (GGE) biplot analysis of multi environmental wheat variety trials. Afr. J. Agric. Res. 8(12):1033-1040.

Bach, S., R.Y. Yada, B. Bizimungu, and J.A. Sullivan. 2012. Genotype by environment interaction effects on fibre components in potato (Solanum tuberosum L.). Euphytica 187(1):7786.

Bonierbale, M., W. Amorós, and J. Espinoza. 2004. Estrategias y desafíos para el mejoramiento de papa para procesamiento. $12 \mathrm{p}$. Agricultura \& Agri-Food, New Brunswick-Canada, CIP Lima, Perú.

Cano, A., and M.B. Arnao. 2004. Actividad antioxidante hidrofílica y lipofílica y contenido en vitamina $C$ en zumos de naranja comerciales: relación con sus características organolépticas. Cienc. Tecnol. Aliment. 4:185-189.

Cotes, J.M., C.E. Nustez, R. Martinez, and N. Estrada. 2002. Analyzing genotype by environment interaction in potato using yield-stability index. American Journal of Potato Research 79(3):211-218

Gedif, M., and D. Yigzaw. 2014. Genotype by environment interaction analysis for tuber yield of potato (Solanum tuberosum L.) using a GGE biplot method in Amhara region, Ethiopia. Agr. Sci. 5:239-249.

Imeh, U., and S. Khokhar. 2002. Distribution of conjugated and free phenols in fruits: Antioxidant activity and cultivar variations. J. Agr. Food Chem. 50:6301-6311.

Iragaba, P. 2014. Inheritance and stability of earliness in potato (Solanum tuberosum L.). Thesis Master in plant breeding and seed systems. College of Postgraduates of Makerere University, Uganda.

Landrum, J. and R. Bone, 2001. Lutein, zeaxanthin and macular pigment. Arch. Biochem. Biophys. 385:28-40.

Lule, D.D., M. Fetene, S. De Villiers, and K. Tesfaye. 2015. Additive main effects and multiplicative interactions (AMMI) and genotype by environment interaction (GGE) biplot analyses aid selection of high yielding and adapted finger millet varieties. J. Appl. Biosci. 76(3):6291-6303.
Maharana, J., C. Panda, and P. Jakhar. 2017. Genotype $\times$ Environment interaction and stability analysis of kharif potato in koraput region of Odisha, India. Int. J. Curr. Microbiol. Appl. Sci. 6(5):1159-1166.

Mohammadi, M., T. Hosseinpour, M. Armion, H. Khanzadeh, and H. Ghojogh. 2016. Analysis of genotype, environment and genotype $x$ environment interaction in bread wheat genotypes using gge biplot. Agri. Commun. $4(3): 1-8$.

Muthoni, J., H. Shimelis, and R. Melis. 2015. Genotype $x$ environment interaction and stability of potato tuber yield and bacterial wilt resistance in Kenya. American Journal of Potato Research 92: 367-378.

Salomón, J.L., J.G. Castillo, J.A. Arzuaga, W. Torres, A. Caballero, M. Varela, and V.M.H. Betancourt. 2015. Analysis of progeny-environment interaction with seedling tubers of true potato seed (Solanum tuberosum L.) in Cuba. Cultivos Tropicales 36(2):83-89.

SAS. 2003. The SAS system for Windows. Release 9.1.3. p. 83-122. SAS Institute Inc., Cary, North Carolina, USA.

Tonk, F.A., E. Ilker, and M. Tosun. 2011. Evaluation of genotype $x$ environment interactions in maize hybrids using GGE biplot analysis. Crop Breed. Appl. Biotechnol. 11:1-9.

Vargas, M., y J. Crossa. 2000. El análisis AMMI y la gráfica del biplot en SAS. 42 p. Unidad de Biometría y Estadística, CIMMYT, México.

Yan, W., and N.A. Tinker. 2006. Analyse par double projection des résultats des essais multi-environnementaux: príncipes et applications. Can. J. Plant Sci. 86:623-645.

Yan, W., L.A. Hunt, Q. Sheng, and Z. Szlavnics. 2000. Cultivar evaluation and mega-environment investigation based on the GGE biplot. Crop Sci. 40:597-605.

Zhang, H., Xu, F., Wu, Y., Hu, H. and Dai, X. 2017. Progress of potato staple food research and industry development in China. J. Integr. Agric. 16(12):2924-2932.

Zobel, R.W., M.S. Wright, and H.G. Gauch. 1988. Statistical analysis of a yield trial. Agron. J. 80:388-393. 